\title{
Review on Epidemiology, Diagnosis, Occupational Hazards and Manage- ment of Pulmonary Tuberculosis in Elderly: A Guide for General Physicians Working in the Health Network Setting, Khuzestan, Iran
}

\author{
Seyed Mohammad Alavi ${ }^{1, *}$, Leila Alavi ${ }^{2}$ \\ ${ }_{1}^{1}$ Medical College, Infectious Diseases department, Jundishapur Infectious and Tropical Diseases Research Center, Jundishapur University of Medical Sciences, Ahvaz, IR Iran \\ 2 Food and Drug deputy of Jundishapur University of Medical Sciences, Ahvaz, IR Iran \\ ${ }^{*}$ Corresponding author: Seyed Mohammad Alavi, Infectious and Tropical Diseases Research Center, Jundishapur University of Medical Sciences, Ahvaz, IR Iran. Tel: +98-611-3387724, \\ Fax:+98-6113337077, E-mail: alavi.seyedmohammad@yahoo.com.
}

Received: Jun 06, 2012; Revised: July 20, 2012; Accepted: August 08, 2012

\begin{abstract}
Elderly, due to immunosuppression caused by predisposing factors such as age, drugs, underlying diseases or malnutrition, is a risk factor for pulmonary tuberculosis (PTB).Aged patients with PTB because of difficulty in diagnosis remain undiagnosed and acts as a source of infection in occupational space of health service units. General physicians (GP) as health care workers (HCW) are concerned about PTB in elderly both as an important public health problem and as an occupational hazard. A systematic review of the literature on the epidemiology, diagnosis, treatment and prevention of tuberculosis in elderly from 1991 to 2012 using computerized bibliographic databases which includes PubMed, Scopus, EMBASE, Google scholar and Iran Medex was carried out to increase understanding of tuberculosis in aged patients attending the health care units. Information obtained from previous studies on PTB has yielded better knowledge about this life threatening illness. This information enables GPs and HCWs to provide improved health services including early diagnosis and treatment of PTB in aged patients as well as prevention of Mycobacterium tuberculosis infection in HCWs at health care units.
\end{abstract}

Keywords: Tuberculosis; Pulmonary; Health Personnel; Elderly

\section{Background}

In recent years, increased frequency of among elderly people worldwide, not only indicates that PTB is an important public health problem but, difficulty in diagnosis and a higher risk of poor outcomes even after treatment completion emphasize the need for specific consideration to this age group (1). According to the World Health Organization (WHO) the proportion ofaged people is growing worldwide, as a result of both longer life expectancy and declining fertility rates (2). The number of old people is growingin Iran chiefly because of improvement of life standards and health services. Population experts estimate that in future 15 years, about 15 percent of the total population of Iran will be 60 years or older (3). In Iran as well as other TB endemic countries, aged people with PTB, were probably infected when young.

Infection with Mycobacterium tuberculosis does not necessarily cause sickness. Those infected may fall ill years later, when they became immunosuppressed. It has been well described that PTB in the elderly is differed from younger patients because of epidemiological factors, delay in diagnosis, adverse reactions to anti TB drugs resultednon-compliance and decreasedcell mediated immunity, thereforeit should be consideredas a separate entity (4). These factors may emerge challenges during Directly Observed Treatment Short Course (DOTS) in the National TB Program (NTP). General physicians, who are concerned with tuberculosis as a major public health, should not overlook the mentioned challenge (5).

\section{Objectives}

The aim of this article is to review the current information about tuberculosis such as epidemiology, diagnosis, treatment, and occupational risks associated in elderly people attending health care workplace.

\section{Search Strategy}

A systematic review of the literature on the epidemiology, diagnosis, treatment and prevention of tuberculosis 
in elderly from 1991 to 2012 using computerized bibliographic databases which includes PubMed, Scopus, EMBASE, Google scholar and IranMedex was carried out to increase understanding of tuberculosis in aged patients attending the health care units.

\section{Elderly and its Trend in the World and in Iran}

As WHO's definition, elderly is referred to every person over 60 years. The proportion of aged people is growing worldwide, as a result of both longer life expectancy and declining fertility rates (2). From 2000 until 2050, the world's population aged 60 and over will be more than triple from 600 million to 2 billion. Most of this increase is occurring in less developed countries, where the number of older people will rise from 400 million in 2000 to 1.7 billion by 2050 (6). According to the latest census which was performed in 2006, the population of elderly people in Iran was 5.123000, which included about 7.5 percent of the general population. It is estimated that in the future of 15 years; more than $15 \%$ of the population of Iran be elderly people (7).

\section{Primary Health Center}

The Primary Health Center (PHC) is the fundamental unit of the public health system providing services throughout Iran, from remote mountain areas to inner urban areas in the country's capital (8). The Iranian Health Care Network provides health services through first line health services called in Iran" Khaneh-e -Behdasht" , health centers and tertiary hospitals $(8,9)$. Since 1984 , the activities of the health system have resulted in a dramatic decrease in the burden of common and endemic infectious diseases $(9,10)$.

\section{Ageing and Infectious Diseases}

Aged individuals due to organ dysfunction and immunologic changes associated with aging are susceptible to infection and are at an increased risk for the incidence and severity of infectious diseases (11). Early diagnosis and treatment is complicated by age-related organ system changes. Because majority of aged people are under treatment with multiple medications for underlying illnesses, drug interactions and adverse events should be kept in mind when antimicrobial therapy needs to be chosen (12). Respiratory tract infections are among the most common infectious diseases in the aged population (13). In both developed and developing countries TB is an important communicable disease which should be considered in aged population (12). Regular use of anti-TB drugs during DOTS program, in the elderly is associated with difficulty due to impaired vision and memory.

\section{Epidemiology}

TB is a major public health problem worldwide. It is es- timated that $M$. tuberculosis has infected one-third of the world's population and 8-9 million new TB cases occur each year (14). TB continues to be a major public health problem in Iran despite the implementation of a national tuberculosis program (NTP); this may be attributed to risk factors such as drug addiction, imprisonment, HIV infection, drug resistant MTB strains and increasing in the number of aged population $(15,16)$. In 1999 the highest and lowest rates of TB in the provinces of Iran were reported to be 137 per 100000 and 10 per 100000 population, respectively, with an average of 26 per 100000 population for the country (17).

Incidence of smear positive pulmonary TB in Khuzestan south west Iran, is estimated to be 25 per 100000 population (18). In another study in Khuzestan, it has been suggested the ageing as a risk factor for pulmonary TB and its mortality (19). In 2008, an estimated 440,000 cases of multi drug resistant (MDR) tuberculosis emerged globally, by 2009 , a total of 58 countries had reported at least one case of extensively drug resistant (XDR) tuberculosis (20). Iran is among countries with reported documented cases with both resistant strains (21). Alavi and colleagues reported MDR-TB as an important cause for TB mortality in Khuzestan especially in aged patients and HIV/AIDS patients, he also estimated that XDR-TB be a major problem against NTP in Khuzestan $(19,21)$.

\section{Risk Factors}

In dealing with elderly people who have chronic cough, considering habits and underlying illnesses such as drug addiction, smoking, diabetes mellitus, chronic renal failure, most of which are the risk factors for TB, helps in early diagnosis of TB. In previous studies the effect of intravenous drug abuse, smoking, diabetes, and corticosteroids in the incidence of tuberculosis has been shown (2126). Aged people with underlying disease, who are mostly under treatment with various drugs, are at increased risk of $\mathrm{TB}(11)$.

\section{Microbiology}

Tuberculosis is caused by M. tuberculosis (MTB). The term acid fast bacillus (AFB) is said to mycobacteria (some other bacteria like nocardia also are acid fast) in ZeihlNeelsen staining (11). In most people, infection with M. tuberculosis is initially defended by cell mediated immunity, and the infection remains latent. However, person with latent infection can be developed into tuberculosis at any time, and become sources of new infections (11). In elderly, tuberculosis is caused by two different ways: 1 ) by a recent infection with $M$. tuberculosis, and, 2) with the reactivation of latent infection years or decades later. Therefore, tuberculosis is defined as" new exogenous infections" or "old endogenous disease".

Reactivation occurs as the result from numerous causes such as poor nutrition status, neoplasia, infection or increasing age. It has been estimated that theannual risk 
of pulmonary TB following a recentinfection is 300 times greater than the risk of illness from reactivation of old disease. Therefore, it has been stated that disease in the elderly largely consists of endogenous reactivation while most tuberculosis in younger people is the result of new exogenous infection (27). However, aged people living in Khuzestan as a TB endemic area with a past history of high incidence rate of TB are very likely to have been infected with MTB and now comprise a growing population group. Aged patients with undiagnosed TB are the main sources for spread of MTB and its resistant strains in occupational places and in the community $(11,25)$.

\subsection{MDR-TB and XDR-TB}

MDR tuberculosis is defined as disease caused by strains of MTB that are at least resistant to isoniazid and rifampicin.XDR-TB is referred to disease caused by MDR-TB that is also resistant to any fluoroquinolone andinjectable second-line anti-tuberculosis drugs such as amikacin, capreomycin, and kanamycin. MDR -TB and XDR-TB have been serious threats to public health worldwide over the past decade (20).

\section{Tuberculin Skin Test}

The tuberculin skin test (TST) is still widely used for the detection of M. tuberculosis infection, although several novel diagnostic assays have been developed in recent years (18). However, TST reactivity may also be observed after bacillus Chalmette-Guerin (BCG) vaccination or mycobacteriumother than tuberculosis (MOTT) infection. Unless vaccination has taken place recently, a TST reaction of greater than $10 \mathrm{~mm}$ should not be attributed to the BCG (18).In aged person due to changes in skin tissues, TST is not a useful test to show latent TB (11).

\section{Clinical Manifestation}

Tuberculosis infection is often asymptomatic but, illness is often associated with general and nonspecific symptoms such as anorexia, weight loss, chills, fever and night sweeting. Depending on the involved organ the organ-related symptoms may also be manifest. In pulmonary $\mathrm{TB}$, symptoms such as chronic cough, bloody sputum and chest pain indicating the spread of the lesion to parietal pleura are also present (11). Although, due to impaired immune function and functional changes, clinical manifestation in the elderly may be different in frequency and severity from younger ones but overally the clinical presentation of pulmonary disease in elderly people does not differ significantly from that in younger patients $(11,28)$.

\section{Diagnosis}

Strong suspicion for the diagnosis of pulmonary TB is usually based on chest X-ray. AFB smear-positive samples helps more in diagnosis, but confirmation of the pulmo- nary TB is made by isolation of M. tuberculosis from sputum culture (11). According to NTP, diagnostic criteria for pulmonary TB is as follow: Cases with at least two sputum smear positive for acid fast bacillus (SSP-AFB) or a chest radiography suggestive of tuberculosis plus one SSP-AFB or, sputum culture positive for $M$. tuberculosis and one SSPAFB, were defined as smear positive pulmonary TB. Cases with clinical finding suggestive of TB plus three sputum smear negative AFB (SSN-AFB) after two weeks of antibiotic therapy plus C-X-ray (suggestive $\mathrm{TB}$ ) were defined as smear negative pulmonary TB (15).

\subsection{Suspected Case}

Any patients with more than 2 weeks of productive cough despite treatment with common antibiotic for respiratory infection and a positive finding in chest X-ray is defined as suspected case of pulmonary $\mathrm{TB}(15)$.

\subsection{Probable Case}

Any suspected case of pulmonary TB with positive results for AFB in sputum according to NTP is defined as Probable case of pulmonary TB (15).

\subsection{Confirmed Case}

Any patient with isolation of $M$. tuberculosis from sputum culture is categorized as confirmed pulmonary TB (15).

\section{Transmission of Tuberculosis in Health Care Workers}

Transmission of TB is a recognized risk to patients and healthcare workers in healthcare settings (30). Transmission is most likely to occur from patients who have unrecognized TB or have received ineffective treatment (31). The risk of transmission of $M$. tuberculosis from patient's toHCWs is a neglected problem in many low and middleincome countries. Most health-care facilities in these countries lack resources to prevent nosocomial transmission of $\mathrm{TB}$ (32). In recent years, the risk of transmission of TB to HCW has attracted minds of health officials (33). Two reasons are suggested for this concern, the first is the resurgence of the disease and the second is the emergence of multidrug-resistant strains of tuberculosis. Cohort studies conducted in the preantibiotic era found a significantly increased risk of disease among health care workers but a study conducted in Hong Kong, reported no excess risk among HCWs, as compared with the risk in the general population (34), whereas another study has reported PTB as a potential occupational hazard (35).

Previous studies in the recent 20 years show that the riskof infection in HCWs is less than 0.2 percent. The transmission happens mostly in high risk HCWs such as nurses, pathology staff, and treating physicians. The main causes of the transmission were: delayed diagnosis of disease, drug-resistant strains and lack of compliance 
with prevention measures such as isolation of patients in isolation rooms with negative pressure ventilation, closing the doors of patient's room and inadequate use of masks by HCWs (36).

\subsection{Source of Infection}

Patients infected with sensitive $M$. tuberculosis strain after 2 weeks treatment according NTP/DOTS, mostly converted to smear negative and became noninfectious but resistant $M$. tuberculosis strains continued their infectivity and were hazardous to HCWs and their contacts (11). Therefore, aged patients with undiagnosed TB are the main sources for spread of M. tuberculosis and its resistant strains in occupational space of health care work places and in the community.

\section{Treatment}

The cornerstone of TB management in National Program against Tuberculosis (NPT) is a standard 6 month course (15) of isoniazid, rifampicin, pyrazinamide and ethambutol, divided in two phases:

1) Intensive phase; The intensive phase included two months treatment with four drugs of isoniazid, rifampicin, pyrazinamide and ethambutol. Drugs are prescribed based on mg per body weight once a day in every morning under supervision of HCW (15).

2) Continue phase; After complement the intensive phase treatment is continued by second phase with isoniazid and rifampicin with the same dose in every morning under supervision of HCW (15).

Aged patients as well as younger patients are recommended to take the adjusted dose of drugs regarding to their renal and hepatic functional changes. Hepatotoxicity which is one of the most serious adverse effects of Isoniazid, pyrazinamide and rifampicin, can lead to such reactions during anti TB therapy, which mostly occurs in older age groups (37). Alavi and colleagues in their work in Khuzestan reported a higher drug induced hepatitis in aged tuberculosis patients (38). In dealing with drug induced hepatitis or other serious drug side effects in elderly patients under anti TB therapy, immediate hospitalization is recommended.

\section{Preventive Measures in Health Care Units}

According WHO guidance the key for TB prevention is stopping the TB at the source, thus the best way to prevent the TB is finding sputum smear-positive PTB patients and cure them until conversion sputum to smear-negative (12). The main causes of the TB transmission in health care units are undiagnosed AFB+ PTB patients particularly aged patients because of delayed diagnosis or misdiagnosis of disease (24). The main key for stopping TB at the source is implantation of DOTS (12). Other prevention measures are as: prevention of drug-resistant strains (17), isolation of patients in isolation rooms with negative pressure ventilation, closing the doors of patient's room and adequate use of masks by HCWs $(24,36)$.

The prevention of TB infection in health-care settings needs all of the following TB-preventive recommendation (39): a) to prevent the generation of droplet nuclei by early diagnosis and treatment of elderly people with active tuberculosis, b) to prevent the spread of infectious droplet nuclei into the air circulation of health care unit space by applying appropriate source-control methods, c) to decrease the number of infectious TB particles in air contaminated with them, c) prescribing appropriate curative and preventive therapy, d) providing isolation rooms for people with, or suspected of having, active PTB, e) screening HCWs for TB infection and tuberculosis, and f) promptly investigating and controlling outbreaks. Previous studies have shown that when inadequate above mentioned preventive measures are done, the risk of TB infection transmission is increased.

\section{Acknowledgements}

None Declared.

\section{Authors' Contribution}

Study concept and design: SM Alavi. Search in literature: SM Alavi, L Alavi. Drafting of the manuscript: SM Alavi. Critical revision of the manuscript for important intellectual content: SM Alavi, L Alavi.

\section{Financial Disclosure}

None Declared.

\section{Funding/Support}

None Declared.

\section{References}

1. Cruz-Hervert LP, Garcia-Garcia L, Ferreyra-Reyes L, Bobadilla-delValle M, Cano-Arellano B, Canizales-Quintero S, et al. Tuberculosis in ageing: high rates, complex diagnosis and poor clinical outcomes. Age Ageing. 2012;41(4):488-95.

2. Ageing. WHO; Available from: http://www.who.int/topics/ageing/en/.

3. Taheri P. [Elderly in Iran]. Health Ministry, Aged population office;Available from: http://www.jamejamonline.ir/.

4. Morris CD. Pulmonary tuberculosis in the elderly: a different disease? Thorax. 1990;45(12):912-3.

5. Moore RM, Jr, Kaczmarek RG. Occupational hazards to health care workers: diverse, ill-defined, and not fully appreciated. Am Jinfect Control.1990;18(5):316-27.

6. What are the public health implications of global ageing? WHO Available from: http://www.who.int/features/qa/42/en/index. html.

7. Esfandiari A. [Aged population and welfare]. Welfare Organization; Available from: http://www.bamdadonline.com/.

8. Lean. 2011; Available from: Available from: http://en.Wikipedia. Org/wiki/Lean.

9. Asadi-Lari M, Sayyari AA, Akbari ME, Gray D. Public health im provement in Iran--lessons from the last 20 years. Public Health. 2004;118(6):395-402.

10. Alavi Seyed Mohammad, Esmaeil Motlagh Mohammad. A Review 
of Epidemiology, Diagnosis and Management of Brucellosis for General Physicians Working in the Iranian Health Network. Jundishapur J Microbiol. 2012;2012(2, Spring):384-387.

11. Fitzgerald DW, Sterling TR, Haas DW, Mandell GL, Bennett JE, Dolin R. Mycobacterium tuberculosis. In: Fitzgerald DW, Sterling TR, Haas DW, Mandell GL, Bennett JE, Dolin R, editors.Principle and Practice of infectious diseases.USA, Philadelphia: Churchill Livingstone; 2010. p. 3129-3163.

12. Mutharayappa R, Bhat TN. Is Lifestyle Influencing Morbidity among Elderly? J Health Manage. 2008;10(2):203-217.

13. Htwe TH, Mushtaq A, Robinson SB, Rosher RB, Khardori N. Infection in the elderly. Infect Dis Clin North Am. 2007;21(3):711-43.

14. Global tuberculosis database. Geneva, Switzerland; WHO. 2008

15. Nasehi National Tuberculosis Program in Iran. Ministry of health, Nashre Seda. 2002.

16. Global tuberculosis control: surveillance, planning, financing. Geneva; WHO. 2005.

17. Epidemiological situation of tuberculosis in Iran. Iran; Ministry of Health. 2002. Alavi SM, Sefidgaran GH. Tuberculin survey among school-aged children in Ahvaz, Iran, 2006. Int J Infect Dis. 2008;12(4):406-9.

18. Alavi SM, Sefidgaran GH. Tuberculin survey among school-aged children in Ahvaz, Iran, 2006. Int J Infect Dis. 2008;12(4):406-9.

19. Alavi SM, Salami Nejad. The causes of death among patients with tuberculosis in Khuzestan, Iran. PakJ Med Sci.2008;24(2):217.

20. Multidrug and extensively drug-resistant TB (M/XDR-TB): 2010 global report on surveillance and response. 2010; Available from: Available from: http://whqlibdoc.who.int/publications/2010/9789241599191_eng.pdf.

21. Alavi SM, Khosravi AD, Seyedian SS. Mycobacterium tuberculosis infection: data based review on Tuberculosis in Khuzestan, Iran. Res J Microbiol. 2011;6(10):724-734.

22. Alavi SM, Sharifi M. Tuberculous spondylitis: risk factors and clinical/paraclinical aspects in the south west of Iran. J Infect Public Health. 2010;3(4):196-200.

23. Alavi SEYED MOHAMMAD, Moola K. Tuberculosis specificity characteristics among febrile patients with systemic lupus erythematosus on steroid therapy. J Gorgan Univ Med Sci. 2010;12(3):Pe79Pe83.

24. Alavi Seyed Mohammad, Ershadian Somayeh. Association between cigarette smoking and pulmonary tuberculosis. J Med Sci. 2009;25(6):912-915

25. Alavi Seyed Mohammad, Farahmand Fatemeh Mohammadi. Pulmonary tuberculosis in household contact of patients with active tuberculosis in Ahwaz, Iran (2003-2005). Pak J Med Sci. 2008;24(6):780-5.

26. Alavi Seyed Mohammad. Relative frequency of infections among hospitalized injecting drug user-HIV positive patients in Razi hospital, Ahvaz, SW Iran (2001-2003). Jundishapur J Microbiol.
2008;2008(1, Winter):6-9.

27. De Backer AI, Mortele KJ, De Keulenaer BL, Parizel PM. Tuberculosis: epidemiology, manifestations, and the value of medical imaging in diagnosis. JBR-BTR. 2006;89(5):243-50.

28. Leung CC, Yew WW, Chan CK, Chau CH, Tam CM, Lam CW, et al Tuberculosis in older people: a retrospective and comparative study from Hong Kong. J Am Geriatr Soc. 2002;50(7):1219-26.

29. Perez-Guzman C, Torres-Cruz A, Villarreal-Velarde H, Vargas MH. Progressive age-related changes in pulmonary tuberculosis images and the effect of diabetes. Am J Respir Crit Care Med. 2000;162(5):1738-40.

30. Morris CD. Pulmonary tuberculosis in the elderly: a different disease? Thorax. 1990;45(12):912-3.

31. Sterling TR, Haas DW. Transmission of Mycobacterium tuberculosis from health care workers. NEngl J Med. 2006;355(2):118-21.

32. Workplace Safety \& Health Topics: Tuberclosis. Available from: Available from: http://www.cdc.gov/niosh/topics/tb/

33. Joshi R, Reingold AL, Menzies D, Pai M. Tuberculosis among health-care workers in low- and middle-income countries: a systematic review. PLoS Med. 2006;3(12).

34. Baussano I, Nunn P, Williams B, Pivetta E, Bugiani M, Scano F. Tuberculosis among health care workers. Emerg Infect Dis. 2011;17(3):488-94

35. Tam CM, Leung CC. Occupational tuberculosis: a review of the literature and the local situation. Hong Kong Med J. 2006;12(6):448-

36. Meredith S, Watson JM, Citron KM, Cockcroft A, Darbyshire JH Are healthcare workers in England and Wales at increased risk of tuberculosis? BMJ.1996;313(7056):522-5.

37. Menzies D, Fanning A, Yuan L, Fitzgerald M. Tuberculosis among health care workers. N Engl J Med.1995;332(2):92-8.

38. Fernandez-Villar A, Sopena B, Fernandez-Villar J, Vazquez-Gallardo $\mathrm{R}$, Ulloa $\mathrm{F}$, Leiro $\mathrm{V}$, et al. The influence of risk factors on the severity of anti-tuberculosis drug-induced hepatotoxicity. Int $J$ Tuberc Lung Dis. 2004;8(12):1499-505.

39. Alavi Seyed Mohammad, Albaji Ali, Sharifi Mehrdad, Tarrahomi Farhad, Zamani Abbas. Drug induced hepatitis among Iranian patients suffering from tuberculosis. PakJ Med Sci.2011;27(2).

40. Guidelines for Preventing the Transmission of Tuberculosis in Health-Care Settings, with Special Focus on HIV-Related Issues. MMWR Recommendation and Reports. 1990 .

Please cite this paper as: Alavi SM, Alavi L. Review on Epidemiology, Diagnosis, Occupational Hazards and Management of Pulmonary Tuberculosis in Elderly: A Guide for General Physicians Working in the Health Network Setting, Khuzestan, Iran. Jundishapur Microbiol. 2013; 6(5): e6677. DOI:10.5812/jjm.6677 